\title{
POLLEN TUBE GROWTH IN CALOTROPIS PROCERA IS CONTROLLED BY ENVIRONMENTAL CHANGES: DOES IT HAVE AN IMPACT ON DELAYED
}

\section{FERTILIZATION?}

\author{
Adina Mishal ${ }^{1,2}$ and Dan Eisikowitch ${ }^{2}$ \\ ${ }^{1}$ Kibbutzim College of Education, Technology and the Arts, Faculty of Sciences, Tel-Aviv, Israel \\ ${ }^{2}$ School of Plant Sciences and Food Security, The George S. Wise Faculty of Life Sciences, Tel Aviv University, Israel
}

Journal of Pollination Ecology,

31(1), 2022, pp 1-7

\section{DOI: $10.26786 / 1920-$ \\ $7603(2022) 659$}

Received 28 July 2021, accepted 16 January 2022

*Corresponding author: dane@tauex.tau.ac.il

\begin{abstract}
Calotropis procera (Apocynaceae) is a Sudanian plant that grows throughout the eastern Saharo-Arabian region. In Israel, it grows along the Rift Valley under extremely hot and dry climatic conditions. In C. procera, as in many other Apocynaceae, the nectar is secreted in the flowers from the nectaries located inside the stigmatic chamber, with the excess flowing via the capillary system into special reservoirs (cucculi). The nectar has two functions: it is used as a reward to attract pollinating insects and it serves as the germination medium for pollen grains. Under natural conditions the nectar concentration is subjected to a large variability, ranging from $22-68 \%$ sucrose. The aim of this study was to elucidate the effects of the natural fluctuations of nectar concentration on pollen germination and pollen tube growth, and their possible role in delaying fertilization in Calotropis procera.

We followed the process of pollen germination under various experimental sucrose concentrations simulating the nectar. We found that the optimal concentration of a sucrose medium for pollen germination is $20 \%$. However, if the alreadygerminated pollen grains are subjected to high sucrose concentration for different periods of time (between one and three hours), elongation of the pollen tubes is inhibited. In all the experimental groups, the pollen tubes renewed their elongation following a reduction of the sucrose. In conclusion, we found that $C$. procera pollen grains' germination is able to adjust to the large fluctuations in sucrose concentration, caused by the changes in temperature and relative humidity conditions of the plant's habitat during the day. This phenomenon probably enables postponing the fertilization towards a time of better conditions and enables the plant to retain the pollen tubes alive, albeit inactive, and thus allow the plant to overcome temporary harsh conditions and develop seeds.
\end{abstract}

Keywords-Apocynaceae, Calotropis procera, environmental stress, nectar, pollen germination, delayed fertilization.

\section{INTRODUCTION}

Calotropis procera (Apple of Sodom) is a Sudanian plant that grows along the Great Rift Valley. In Israel it occurs mainly in the area surrounding the Dead Sea, reaching northwards as far as the Sea of Galilee and southwards as far as Eilat, where it grows near water sources under extreme climate conditions of high heat and low humidity (Eisikowitch 1986; Zohary \& Feinbrun 1966). Florescence takes place between March and September (Eisikowitch 1986). In C. procera, as in many other Asclepiadoideae (Apocynaceae), nectar is produced in the nectaries, located inside the walls of the stigmatic chambers (Galil \& Zeroni 1965, 1969).

The morphology of C. procera flowers is similar to that of Asclepias curassavica (Mexican butterfly weed, Galil \& Zeroni 1965) and found to be highly complex (Fig. 1). Longitudinal sectioning reveals a superior ovary composed of two separate ovarian lobes, with only the lower part of the tissue common to both. This is the external exposed part. The precise location of the receptive part of the 
stigma is still unknown, but it is clear that is not external, as described at Ali \& Ali (1989).

Each anther has two pollen sacs, with each sac enclosing a single pollen chamber. The pollen grains that develop in the pollen chamber remain as a single mass bound by a rigid membrane with germination slots. This mass, the pollinium, functions as a single pollen grain that develops multiple pollen tubes.
Each pair of pollinia is linked by a sticky substance that hardens and binds together each adjacent pair. The link is secured by a sticky pincer connected to the pollinia by two branches (Fig. 1B). The five stamens are fused together at the base where they surround the styles, while being separated by tightly closed slots at the perimeter to form five narrow "stigmatic chambers" (Fig. 2G) Galil \& Zeroni (1965) .
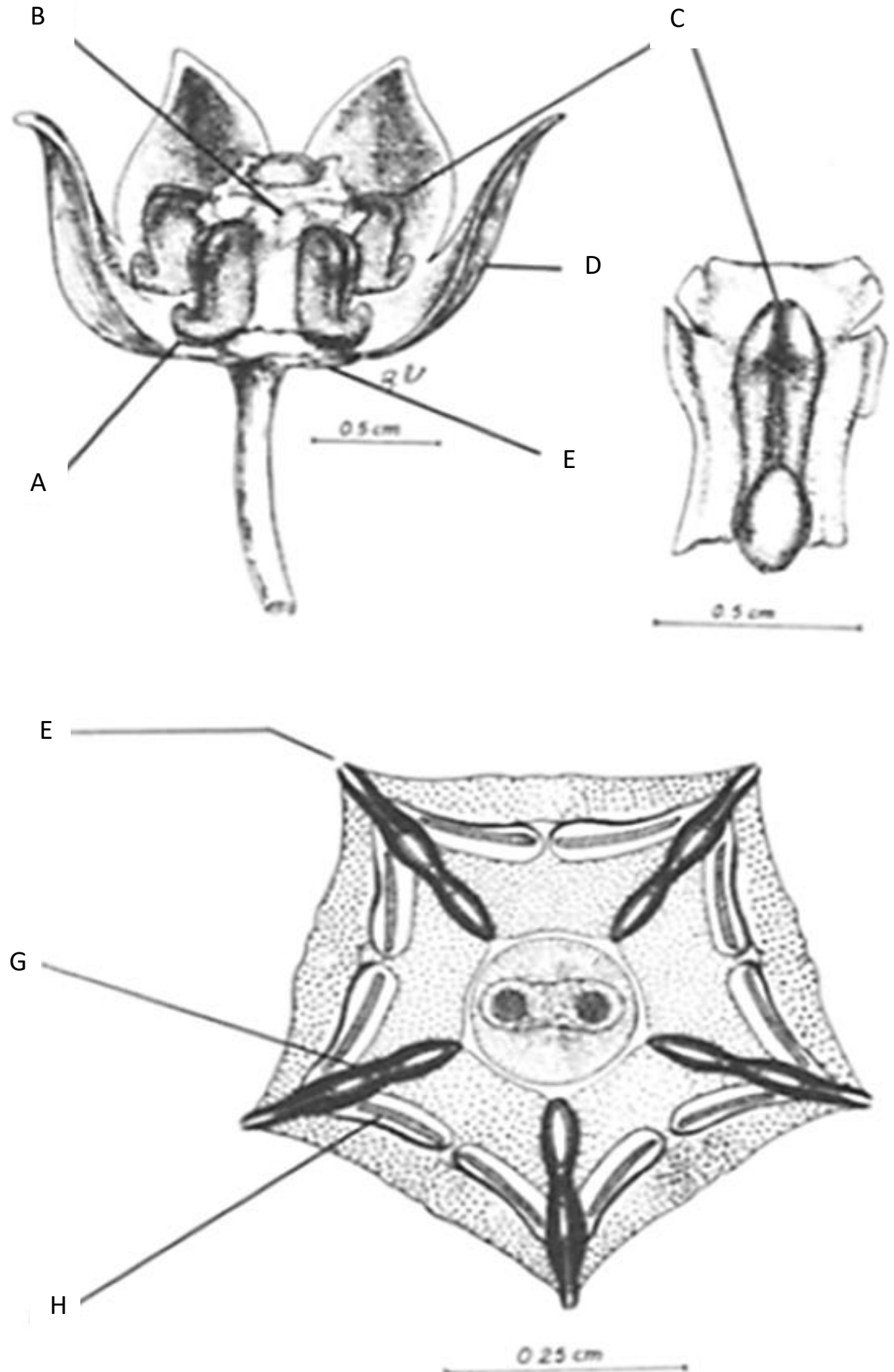

Figure 1: A lateral view of a C. procera flower. A. cucculus; B. corpusculum; C. entrance to the cucculus; D. petal; E. sepal (Eisikowitch 1986).

Figure 2: A cross-section of a C. procera flower. $F$. entrance to the stigmatic chamber; $G$. stigmatic chamber; H. pollinium (Eisikowitch 1986). 
The inner walls of these stigmatic chambers are lined with glandular tissue that secretes the flower's nectar, which is then conducted via ducts to receptacles - the cucculi - located opposite the stigmatic chambers. These protruding receptacles, which surround the gynostegium like a corona, are composed of thick, hard tissue, and each has an opening directed upwards - an extremely narrow slot protected by dense hairs (Fig. 1C).

The nectar produced in the stigmatic chambers is thus conducted to the cucculi, while remaining in an almost entirely closed system throughout the process. It is only accessible via the slots in the stigmatic chambers (Fig. 2F) or through the entry slots of the nectar receptacles - the cucculi (Fig 1C).

In Israel, $C$. procera is pollinated by two carpenter bees of the genus Xylocopa (Eisikowitch 1986; Eisikowitch 1987; Gerling et al. 1982). In order to collect the nectar, the bee must insert its proboscis through the slots into the cucculi in which the nectar is stored. The anterior sides of the stigmatic chamber enclose the pairs of pollinia, which adhere to the legs of the bees and are extracted from the flower when the bees withdraw their legs. The pollinia then become inserted into the stigmatic chamber when the bees attempt to gather nectar from another flower. The pollen grains in the pollinium germinate in the nectar that fills the stigmatic chamber, rather than on a specific stigma, as is typical of flowering plants. The nectar thus serves both as a reward for pollinators and as a substrate for pollen germination (see also Broyles 2019).

In field observations at Hazeva (Eisikowitch 1986) nectar concentration in C. procera was found to fluctuate throughout the day. Its concentration, in equivalence to that of sucrose, ranged between $22 \%$ and $68 \%$, with bees visiting throughout the day (Fig. 3). Similar findings were reported in the study carried out between April and September by Dagan (1994). This raises several questions concerning the pollination and fertilization process in C. procera.

Since pollen germination takes place in the nectar, and since nectar concentration ranges from $22 \%$ to $68 \%$, this raises the question of how the reproductive mechanism in C. procera is adapted to cope with these fluctuations: in other words, how do the natural fluctuations in nectar concentration affect pollinium germination and pollen tube growth?

The aim of this study was to elucidate the effects of the natural fluctuations of nectar concentration on pollen germination and pollen tube growth, and its possible role in delaying fertilization in Calotropis procera.

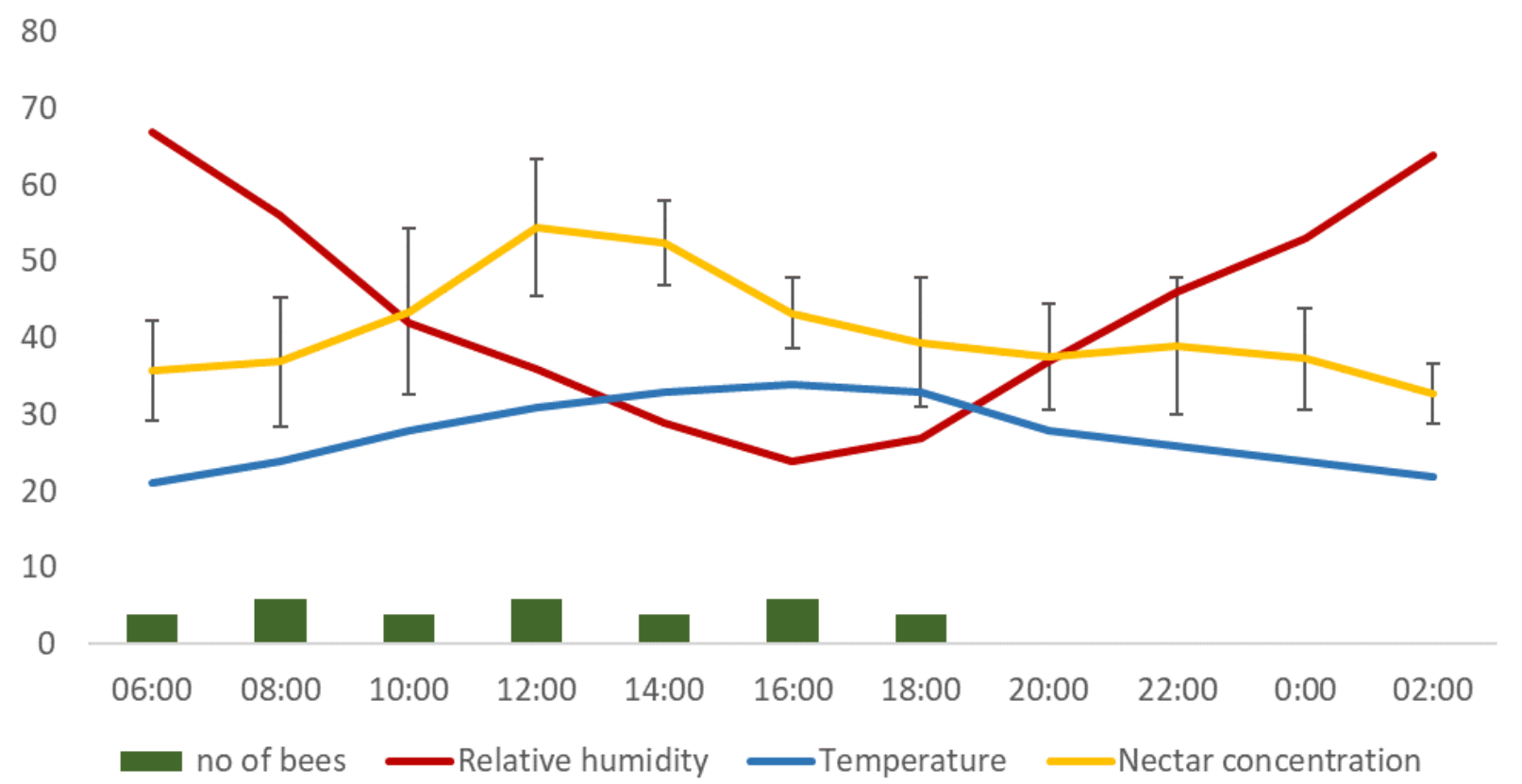

Figure 3: Fluctuations in nectar concentration (\%), relative humidity (\%) and temperature $\left(\mathrm{C}^{\circ}\right)$ over 24 hrs, in relation to bee activity; adapted from Eisikowitch (1986). 


\section{MATERIALS AND METHODS}

In order to determine the optimal sucrose concentration for in-vitro pollinium germination, pollinia were first germinated for two hours at sucrose concentrations ranging from $10 \%$ to $50 \%$. Following determination of the optimal sucrose concentration, the pollinia were then placed in the optimal solution for seven hours, while monitoring pollen tube length. During this latter stage, we had already determined that five hours were sufficient for optimal pollen tube length. In the third stage, we transferred the pollinia to the optimal solution for five hours, and then to an extreme sucrose concentration (50\%) for varying lengths of time before finally returning them to the optimal concentration.

1. Preparation of the germination solution: a solution of $200 \mu \mathrm{g} / \mathrm{ml}$ boric acid $\left(\mathrm{H}_{3} \mathrm{BO}_{3}\right)$. This constitutes the basis for preparing germination solutions at varying sucrose concentrations (Dagan 1994).

2. Monitoring pollen tube germination: germination is considered to have occurred when the length of the pollen tube equals the width of the pollinium.

3. Vigour of germination was scored following Kevan et al. (1989): Level 1 - pollen tube length less than half width of pollinium; Level 2 - pollen tube length between half and entire width of pollinium; Level 3 - pollen tube length greater than $100 \%$ but less than $150 \%$ of pollinium width; and Level 4 - pollen tube length $150 \%$ or more of pollinium width.

\section{EXPERIMENTS}

Pollinia from several plants $(N=40$ in each group) were germinated in a standard solution of $20 \%$ sucrose for five hours. They were then transferred to a solution of $50 \%$ sucrose for the following periods of time:

experimental group 1 - one hour

experimental group 2 - two hours

experimental group 3 - three hours

control group A - remained in 20\% sucrose solution (not transferred to $50 \%$ solution)

control group B - transferred to 50\% sucrose solution for 16 hours (not returned to $20 \%$ solution)

Pollinia in the three experimental groups were returned to the $20 \%$ solution after the predetermined interval, and the growth of the pollen tubes was then monitored for the following 16 hours. Growth was recorded according to germination vigour levels ( 1 through 4 ) as defined in section 3 above.

\section{RESULtS}

The highest percentage of pollen tube germination (90\%) occurred in the $20 \%$ sucrose germination solution (Fig. 4).

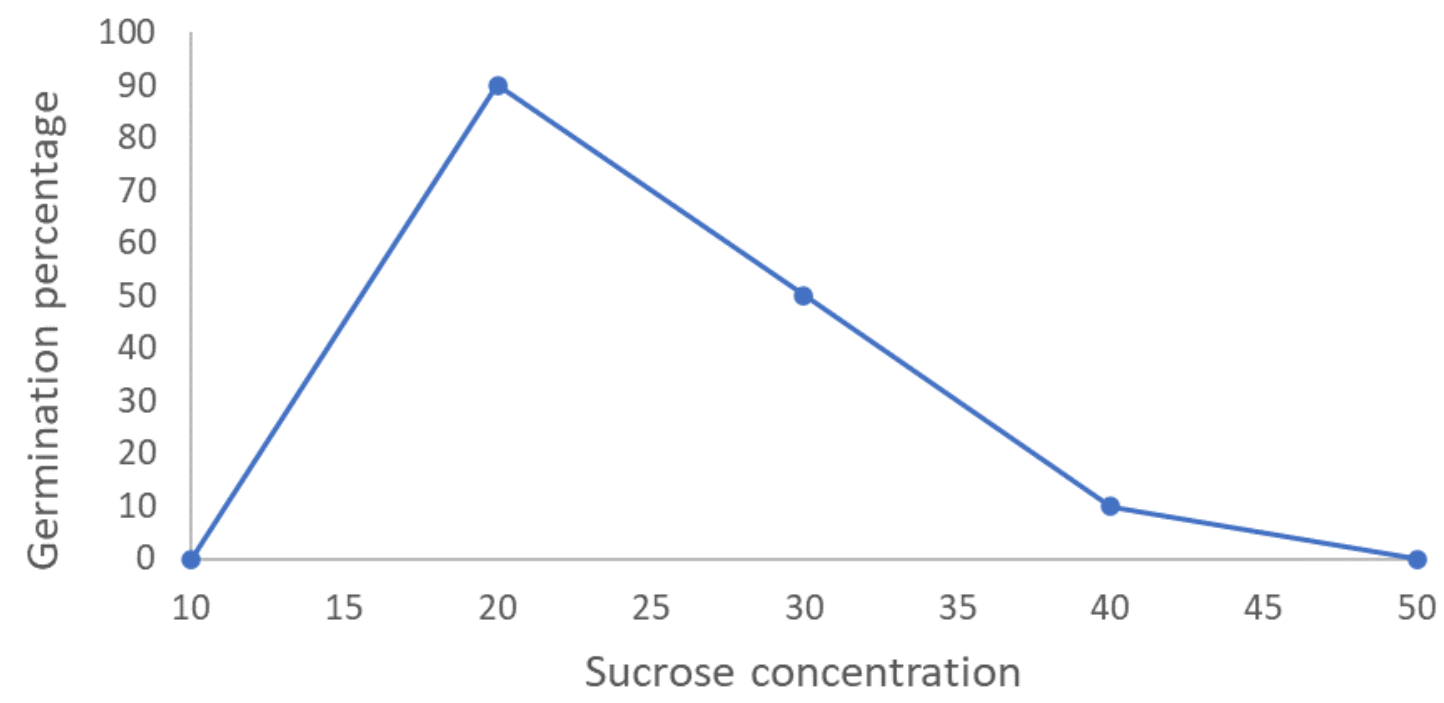

Figure 4: Effect of sucrose concentration on rate of germination ( $N=40$ in each group) 


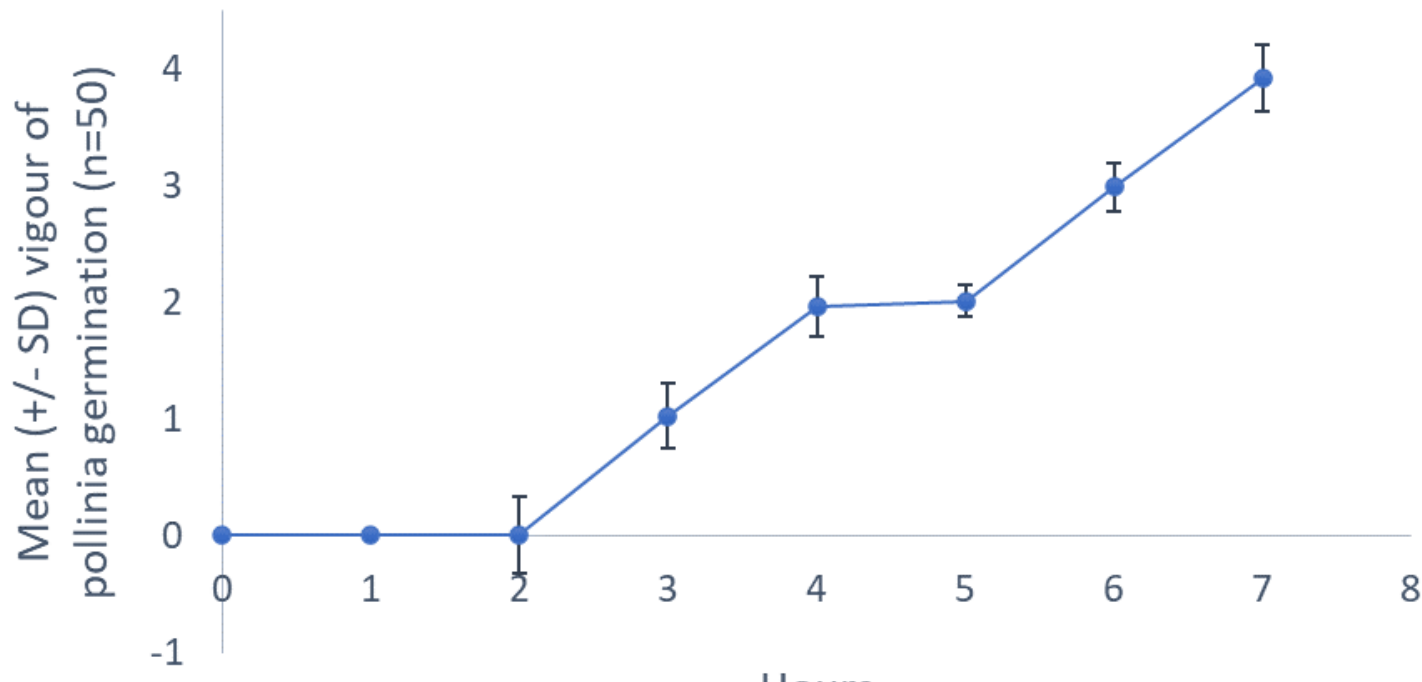

Hours

Figure 5: Vigour of germination (levels 1-4) over time in a $20 \%$ sucrose solution (S.D)

Fig. 5 presents pollen germinated in a $20 \%$ sucrose solution. Germination solution reached level 1 after 3 hours, level 2 after 4 hours, level 3 after 6 hours and level 4 after 7 hours.

As shown in Fig. 6, when pollen grains were germinated in a $20 \%$ sucrose solution under optimal conditions for five hours, then transferred to the extreme conditions of a $50 \%$ sucrose solution, the pollen tubes stopped growing. However, even following three hours under extreme conditions, when they were returned to optimal sucrose solution the pollen tubes resumed lengthening within several hours.

\section{Discussion}

Nectar concentration is influenced by environmental factors such as temperature and humidity (see Fig. 3). Since an essential part of the

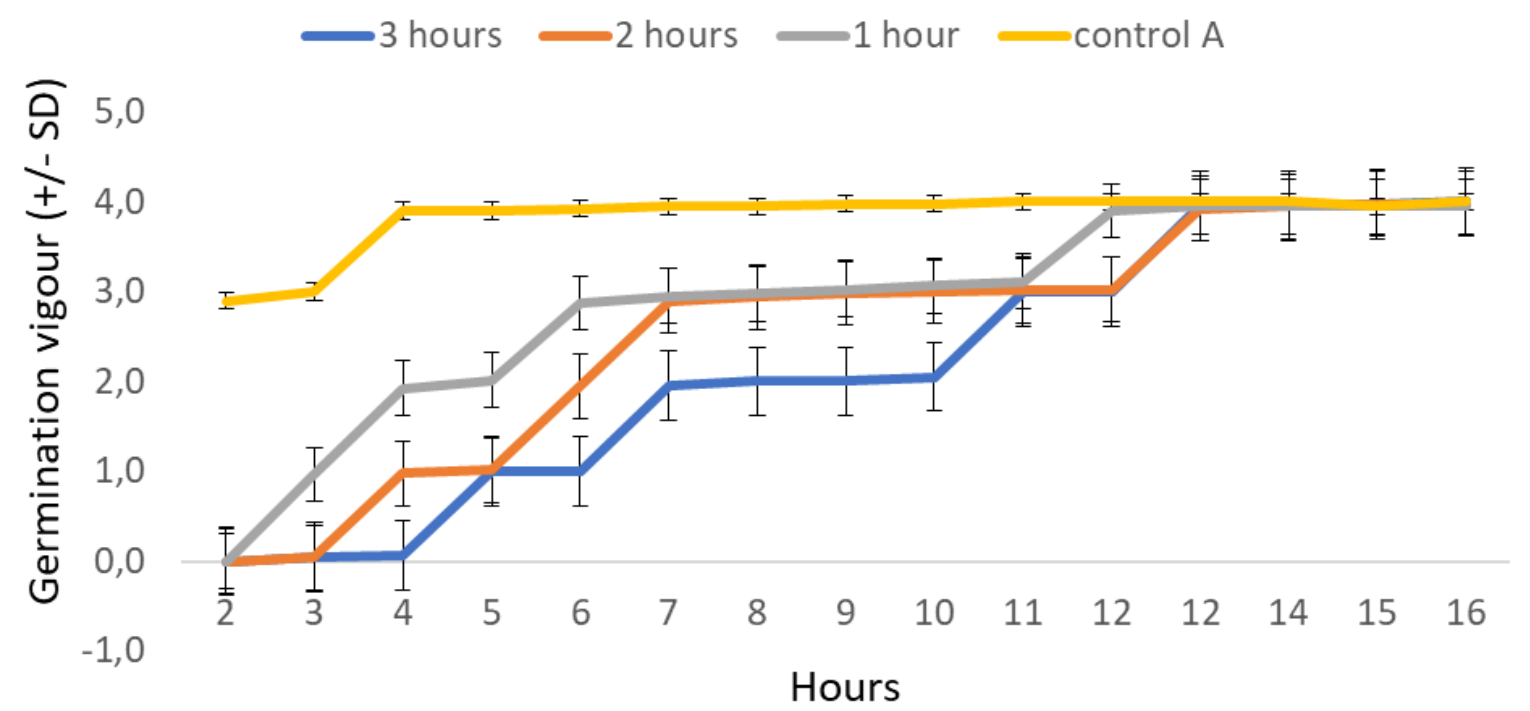

Figure 6: Germination vigour (means) (levels 1-4) of germinated pollen grains returned to a $20 \%$ sucrose solution after being placed in a $50 \%$ solution for varying periods of time $(N=40)$ 
reproductive process of Calotropis procera takes place in the nectar, variations in the nectar concentration directly affect this process (see Fig. 4).

The findings of this study lead to the conclusion that $C$. procera is well-adapted to contending with extreme climate conditions of heat and aridity. These conditions lead to increasing nectar concentration over the course of a day. Since the nectar in the cucculi is connected via capillary system with the stigmatic chamber, it means that when nectar concentration rises in the flower cuculi, the germination process of the pollen tubes inside the nectar of the stigmatic chamber is halted, and this state of suspended germination may continue for several hours. When the temperature drops and relative humidity rises nectar concentration decreases, the germination process continues from the point at which it was interrupted.

In a review of the existing literature, we found that Kevan et al. (1989) indicated that high nectar concentration might cease pollen elongation, However Broyles \& Stoj (2019) mentioned in their abstract that "pollen germination could be inhibited when the sugar concentration increases", and in their discussion they stated that even when nectar concentration is elevated above $50 \%$ during the day, nectar holding deep in the flower has an optimal concentration for pollen germination. They did not measure nectar concentration within the stigmatic chamber, and neither did we. Since we are dealing with an almost closed capillary system, we suppose that nectar concentration is equalized and brings the stigmatic chamber and the cucculi to the same concentration.

Here we concluded that the reproduction process is suspended and renewed at the pollen tube germination stage by the level of nectar fluctuations. In certain gymnosperms, such as Pinus monticola (Pinaceae) and Agathis australis (Araucariaceae), a development time of approximately one year from pollination to seed maturation has been reported (Owens et al. 1995; Owens \& Bruns 2000). However, these are cases in which the period of development is fixed for each species and applies to all the seeds uniformly. A study on the effects of stress on Jatropha curcas $L$. also found a singular phenomenon whereby the development of fruit is delayed and sometimes prolonged for several months, until the fruit has fully matured (Samocha 2012). Similar phenomena have been described in animals. Thus, for instance, when fertilized females of certain insects underwent experimental starvation, some of the embryos were resorbed, while the development of others either continued or was suspended (Ward and Dixon, 1982). A study of roe deer revealed a phenomenon of delayed implantation in cases in which environmental conditions restricted nutrition. In such cases the fertilized ova remained in the uterus for approximately five months before implantation (Aitken 1974; Sandell 1990).

In this study we observed that in Calotropis procera, under stressful environmental conditions in which nectar concentration rises above a certain threshold, pollen grains' germination is temporarily halted, and re-activated as these conditions improve. We suggest that this ability to adjust to large fluctuations in sucrose concentration following the changes in temperature and relative humidity conditions of the plant's habitat, may have evolved in order to enable the plant to retain the pollen tubes alive, albeit inactive, and thus allow the plant to overcome daily episodes of harsh conditions, and develop seeds.

\section{REFERENCES}

Aitken RJ (1974) Delayed implantation in roe deer (Capreolus capreolus). Reproduction, 39 (1):225-233.

Ali T, Ali SI (1989) Pollination biology of Calotropis procera subsp. hamiltonii (Asclepiadaceae). Phyton (Austria) 29:175-188.

Broyles S (2019) Patterns of Nectar Production in Asclepias curassavica (Apocynaceae). Journal of Pollination Ecology, 25:78-88.

Dagan R (1994) Dynamics of pollen grain germination in Calotropis procera (Aiton) R. Br., MSc dissertation, Tel Aviv University.

Eisikowitch D (1986) Morpho-ecological aspects on the pollination of Calotropis procera (Asclepiadaceae) in Israel. Plant Systematics and Evolution, 152 (3):185194.

Eisikowitch D (1987). Calotropis procera (Ait.) Ait. F. (Asclepiadaceae) and Xylocopa spp.: a study of interrelationships. Series Entomologic:341 - 345.

Galil J, Zeroni M (1965) Nectar system of Asclepias curassavica. Botanical Gazette, 126 (2): 144-148. 
Galil J, Zeroni M (1969) On the organization of the pollinium in Asclepias curassavica. Botanical Gazette, 130 (1):1-4.

Gerling D, Hurd Jr PD, Hefetz A (1982) Comparative behavioral biology of two Middle East species of carpenter bees (Xylocopa latreille) (Hymenoptera: Apoidea). Smithsonian Contributions to Zoology.

Kevan PG, Eisikowitch D, Rathwell B (1989) The Role of Nectar in the Germination of Pollen in Asclepias syriaca L. Botanical Gazette, 150 (3):266-270.

Owens JN, Bruns D (2000) Western white pine (Pinus monticola Dougl.) reproduction: I. Gametophyte development. Sexual Plant Reproduction, 13 (2):61-74.

Owens JN, Catalano GL, Morris SJ, Aitken-Christie J (1995) The reproductive biology of Kauri (Agathis australis). I. Pollination and pre-fertilization development. International Journal of Plant Sciences, 156 (3):257-269.

Samocha Y (2012) Pollination and fertilization biology of Jatropha curcas $L$. and its implications for oil yield under Mediterranean conditions, $\mathrm{PhD}$ dissertation, Tel Aviv University.

Sandell M (1990) The evolution of seasonal delayed implantation. The Quarterly Review of Biology, 65 (1):23-42.

Ward SA, Dixon AF G (1982) Selective resorption of aphid embryos and habitat changes relative to lifespan. The Journal of Animal Ecology:859-864.

Zohary M, Feinbrun-Dothan N (1966) Flora Palaestina, Calotropis procera pt.1. Jerusalem: Israel Academy of Sciences and Humanities. 